\title{
Hybrid quantum dot-based theranostic nanomedicines for tumor-targeted drug delivery and cancer imaging
}

\author{
Dina G Zayed ${ }^{\ddagger 1,2}$, Ahmed S AbdElhamid $¥, 1,3$, May S Freag ${ }^{1,4,5,6}$ \& Ahmed O Elzoghby*,1,2,5,6 \\ ${ }^{1}$ Cancer Nanotechnology Research Laboratory (CNRL), Faculty of Pharmacy, Alexandria University, Alexandria 21521, Egypt \\ ${ }^{2}$ Department of Industrial Pharmacy, Faculty of Pharmacy, Alexandria University, Alexandria 21521, Egypt \\ ${ }^{3}$ Department of Pharmaceutical Technology, Faculty of Pharmacy, Tanta University, Tanta, Egypt \\ ${ }^{4}$ Department of Pharmaceutics, Faculty of Pharmacy, Alexandria University, Alexandria 21521, Egypt \\ ${ }^{5}$ Division of Engineering in Medicine, Department of Medicine, Brigham \& Women's Hospital, Harvard Medical School, Boston, MA \\ 02115, USA \\ ${ }^{6}$ Harvard-MIT Division of Health Sciences \& Technology (HST), Cambridge, MA 02139, USA \\ *Author for correspondence: aelzoghby@bwh.harvard.edu \\ ${ }^{\ddagger}$ Authors contributed equally
}

First draft submitted: 28 October 2018; Accepted for publication: 22 November 2018; Published online: 17 January 2019

Keywords: cancer theranostics $\bullet$ drug delivery $\bullet$ inorganic nanoparticles $\bullet$ lipids $\bullet$ nanohybrids $\bullet$ polymers $\bullet$ polysaccharides $\bullet$ proteins $\bullet$ quantum dots $\bullet$ tumor targeting

\section{Quantum dots \& role in cancer imaging}

Over the last decade, researchers have paid great interest to multimodal drug-delivery systems. Quantum dots (QDs) are considered one of the most efficient tools used in theranostic applications for diagnosis and therapy due to their unique physiochemical characteristics. QDs are semiconductor crystals in the nano-scale range of 2-10 nm, which exhibit unique photoluminescence characteristics as well as electronic properties such as tunable emission from visible to near infrared wavelengths and superior light stability [1]. QDs possess strong photoluminescence with high molar extinction coefficient values, which make them the best candidates for cell labeling and detection of cancer biomarkers. QDs are characterized by symmetric narrow-emission spectra and broad-absorption spectra [1,2]. However, some fears have been raised regarding the toxicity of QDs, especially Cd-containing QDs, due to the release of $\mathrm{Cd}$ ions and the generation of reactive oxygen species [2]. Therefore, strategies have been developed to reduce their toxicity and enhance their biocompatibility through hybridization with other moieties such as polymers, proteins, polysaccharides or lipids, offering efficient tumor targeting in addition to inhibiting their release into the systemic circulation. This article discusses QD-based nanohybrids for delivery of anticancer drugs in combination with cancer imaging.

\section{Protein-QD hybrid nano-theranostics}

Protein-QD nanohybrids exhibiting high stability in biological fluids play a crucial role in bioimaging applications. QDs can be hybridized with proteins via either chemical coupling or physical entrapment. For example, graphene QDs have been successfully conjugated to the surface of gemcitabine-loaded human serum albumin nanoparticles (NPs) [3]. In this study, by Nigam et al. a strong green fluorescence was observed inside Panc-1 cells treated with the NPs, demonstrating their efficient uptake in tumor cells. On the other hand, encapsulation of CdSe QDs into gelatin NPs has been found to improve QD biocompatibility where QD-gelatin NPs exhibited no toxic effect on cells up to a concentration of $5 \mathrm{mg} / \mathrm{ml}$ while maintaining their strong fluorescent properties [4]. In another study, multi-fluorescent nanospheres were developed by physical encapsulation of different-sized CdTe/CdS QDs together into single bovine serum albumin (BSA) nanospheres via spray-drying [5]. The fluorescence of nanospheres could be modified by changing the size of the QDs. The typical fluorescence stabilities of the QD-BSA nanospheres were studied and it was found that, when the QD-BSA nano-spheres contained a high molar ratio of QD:BSA, the fluorescence emission decreased by $4.06 \%$ after being continuously irradiated (at $365 \mathrm{~nm}$ ) for $1 \mathrm{~h}$. These results

Future 8 Medicine 
revealed that the fluorescence of the QD-BSA nanospheres could be used for long-term fluorescence observation in biomedical research areas.

Zein-ZnS QD nanohybrids could be utilized for drug delivery of compounds such as 5-fluorouracil. In order to determine the biocompatibility of these nanohybrids, Aswathy et al. investigated their effect on the viability of L929 and MCF-7 cancer cells [6]. The cell viability was found to be more than 90 and $80 \%$ for plain zein nanoparticles and zein-QDs, respectively, which indicated their compatibility. However, when treated with 5-FU-loaded zein-QDs, the cell viability was significantly reduced. Moreover, the emitted fluorescence of the zein-ZnS QD NPs indicated their successful internalization into the cells. Among other interesting approaches to enhance tumor penetration, one study fabricated multistage QD nanocarriers by conjugating silica-coated QDs to the surface of gelatin NPs forming $100 \mathrm{~nm}$ nanohybrids [7]. After extravasation into tumor tissue, the gelatin core was hydrolyzed by the upregulated matrix metalloproteinases in the tumor microenvironment, thus releasing ultra-small $10 \mathrm{~nm}$ QDs, allowing efficient penetration into the tumor parenchyma.

Overall, using proteins for hybridization of QDs can be a potential strategy to prolong their systemic circulation, increase their physical stability, enhance their tumor targetability and reduce their toxicity.

\section{Polysaccharide-QD hybrid nano-theranostics}

Different types of polysaccharide can be utilized to improve safety and biocompatibility of QDs in order to enable nontoxic imaging. Incorporation of $\mathrm{Mn}: \mathrm{ZnS}$ QDs within chitosan can be utilized to reduce its toxicity and enable functionalization with folic acid. Indeed, Bwatanglang et al. combined the targeted drug delivery and imaging capabilities of folic acid (FA) and Mn-doped ZnS QDs (Mn-ZnS QDs), respectively, in a single nano delivery system [8]. FA-chitosan conjugate (FACS) was prepared, then electrostatically complexed with Mn-ZnS QDs forming FACS-Mn-ZnS nanocomposite. Both bare Mn:ZnS QDs and FACS-Mn:ZnS (7-500 $\mu \mathrm{g} / \mathrm{ml})$ did not exhibit toxicity against breast cancer cells (MCF-7 and MDA-MB-231) and normal human breast cells (MCF-10) after $24 \mathrm{~h}$. By increasing the QDs concentration from 62 to $500 \mu \mathrm{g} / \mathrm{ml}$, only a slight reduction in the viability of cells treated with FACS-Mn:ZnS compared with those exposed to bare Mn:ZnS QDs was observed. This slight reduction in cell viability for the cells treated with FACS-Mn: $\mathrm{ZnS}$ could be correlated to the enhanced binding between the FA-coupled QDs with the folate receptor-expressing cancer cells. Moreover, the FA-conjugated QDs emitted stronger cellular fluorescence upon attachment to the folate receptors expressed on the cancer cells compared with milder fluorescence observed in cells treated with nontargeted Mn-ZnS QDs.

Coupling of QDs with hyaluronic acid has been found to be an interesting tool for enhanced intracellular delivery into liver cells, mediated via interaction with CD44-receptors, thus enabling in vivo real-time visualization $[9,10]$. In our laboratory, $\mathrm{CdTe}$ QD theranostic nanocapsules were developed in which the anionic polysaccharide chondroitin sulfate was used to coat the positively charged oily core co-loaded with rapamycin and celecoxib [11]. An outer layer of cationic gelatin-coupled QDs was deposited onto chondroitin sulfate nanocapsules to inhibit their nonspecific uptake by normal cells. At tumor sites, gelatin was hydrolyzed by matrix metalloproteinases releasing both drug nanocapsules and QDs into cancer cells for imaging. In a parallel study, replacing gelatin with the iron-binding cationic protein, lactoferrin resulted in an ON-OFF effect where the fluorescence of QDs was initially quenched by energy transfer which was then restored after bond cleavage in tumor cells [12]. Thus, the in vitro and in vivo localization of nanocapsules into breast tumors was visualized utilizing QDs fluorescence.

Based on the above-mentioned studies, it can be seen that hybridization of QDs with polysaccharides can improve its tumor targeting capability via binding to their receptors overexpressed by cancer cells, and hence increase their accumulation at the tumor site.

\section{Polymer-QD hybrid nano-theranostics}

One of the most important approaches for QD surface functionalization is their encapsulation into a layer of amphiphilic diblock or triblock copolymer. Volsi et al. [13] functionalized $\alpha, \beta$-poly $(N$-hydroxyethyl)-D,L-aspartamide (PHEA) with lipoic acid (LA), PEG and FA to form an amphiphilic copolymer (PHEA-LA-PEG-FA) that selfassembled into micelles in aqueous medium. Hybrid gold-silica/CdSe-CdS QDs (Au-SiO $\left.{ }_{2} / \mathrm{QDs}\right)$ were prepared and co-encapsulated with doxorubicin into the hydrophobic core of PHEA-LA-PEG-FA micelles. The fluorescent nanoprobes demonstrated high internalization into MCF-7 breast cancer cells and localized mainly in the cytoplasm. Upon irradiation of MCF-7 cells with a laser beam at $810 \mathrm{~nm}, \mathrm{Au}-\mathrm{SiO}_{2} / \mathrm{QDs}$ micelles demonstrated more powerful cytotoxic effect to the cells compared with free doxorubicin, which could be correlated to the hyperthermic 
effect of $\mathrm{Au}-\mathrm{SiO}_{2} / \mathrm{QDs}$. A synergistic photothermal/chemotherapeutic killing effect was obtained when cells were irradiated in the presence of both QDs and doxorubicin co-loaded micelles [13].

Similarly, the near-infrared InP/ZnS QDs were functionalized with the amphiphilic polylactide-b-polyethylene glycol (PLA-PEG) copolymer resulting in QD-based micelles loaded with the anticancer drug, aminoflavone [14]. Anti-EGFR nanobodies were conjugated to the surface of the micelles to target EGFR-overexpressing cancer cells. The QD-based theranostic micelles led to tumor regression in an orthotopic EGFR-overexpressing triple negative breast cancer mouse model. Conclusively, hybridization of fluorescent QDs with polymers can offer promising characteristics including easy surface functionalization and enhanced colloidal stability. Furthermore, research studies should be conducted on the toxicity and biodistribution profiles of such nanohybrids.

\section{Lipid-QD hybrid nano-theranostics}

Lipid-QD hybridization can offer many advantages and decreased toxicity of QDs through various approaches. For example, Olerile et al. co-loaded paclitaxel with $\mathrm{CdTe} / \mathrm{CdS} / \mathrm{ZnS}$ QDs into nanostructured lipid carriers (NLCs) composed of glyceryl monostearate, soya phosphatidylcholine and oleic acid as a theranostic modality in order to form a splendid parenteral drug delivery system to enhance the efficacy of paclitaxel and the ability to detect and image tumors [15]. Near-infrared fluorescence imaging demonstrated that the NLCs accumulated efficiently in liver cancer cells of mice as tracked via QD fluorescence. Moreover, a remarkable degree of safety was confirmed through histopathological assessment. Tissues treated with both control and co-loaded NLCs showed relatively no significant cytoarchitectural differences or toxicity in different organs. In addition to NLCs, hydrophobic QDs can be encapsulated within the lipid bilayers of liposomes to form nanoscale vesicles [16]. Thus, higher compatibility of QDs with aqueous media as well as enhanced photostability could be guaranteed. Upon pharmacokinetic analysis, cationic liposome-QDs nanohybrids significantly accumulated in lung and kidney. In another study, hybrid QD-immunoliposomes exhibited good imaging and glioblastoma treatment through efficient uptake by malignant cells [17]. CdSeTe/ZnS core/shell QDs were conjugated to PEGylated lipids on liposomal surfaces to serve as luminescent reporters. These reporters were utilized to detect QD-immunoliposome fluorescent signals to demonstrate their infusion by convection-enhanced delivery into intracranial tumors. These hybrids exhibited an efficient in vitro uptake and 1.3- to 5.0-fold higher total fluorescence in intracranial brain tumor xenografts in vivo compared with a nontargeted counterpart [17]. Overall, incorporation of QDs into lipid nanocarriers was found to improve their biocompatibility and safety profile, thus paving the way for their possible clinical translation.

\section{Inorganic-QD nanohybrid theranostics}

The hybridization between gold NPs and QDs can result in multifunctional nanohybrids which enable both superior imaging and anticancer activity. Chen $e$ al. developed $\mathrm{ZnO}$ QDs conjugated to gold NPs loaded with the anticancer drug camptothecin [18]. As gold NPs can destroy tumor cells via photo-thermal therapy by converting the absorbed light energy into localized heat, both blank and drug-loaded nanocarriers exhibited powerful cytotoxicity toward HeLa cells. On the other hand, dual modal imaging modality could be achieved by hybridization between paramagnetic Gd ion and CuInS/ZnSQDs to enable dual fluorescence/magnetic resonance-mediated diagnosis. Yang et al. prepared Cd-free Gd-doped ZnS QDs in lipid vesicles with optimized fluorescence and good colloidal stability properties [19]. Similarly, super-paramagnetic $\mathrm{Fe}_{3} \mathrm{O}_{4}$ were separated from the fluorescent graphene-CdTe QDs by $\mathrm{SiO}_{2}$ shell to avoid fluorescence quenching of QDs [20]. This bi-functional cyto-compatible model allowed high loading of 5-fluorouracil and was effective against hepatoma cells. Overall, hybridization of QDs with multiple types of inorganic NPs can enable their use for multi-modal imaging, for example, fluorescence, magnetic or ultrasound imaging. In addition, combined therapeutic modalities could be achieved for synergistic cancer therapy, for example, magnetic hyperthermia, photothermal and photodynamic therapy.

\section{Challenges \& future perspective}

QDs have several powerful advantages in bioimaging over conventional dyes which enable QDs to attract tremendous interest in various biological fields. However, there are many challenges facing these applications, such as cellular toxicity due to reactive oxygen species generation and cadmium release (for cadmium-containing QDs). In this article, we reported different techniques used to alleviate QDs toxicity via hybridization with different biocompatible polymers, proteins, lipids or inorganic nanoparticles and discussed the invention of different multifunctional platforms for cancer therapy and diagnosis. We claim that these approaches were well designed to overcome many challenges facing theranostic applications. 
Financial \& competing interests disclosure

The authors have no relevant affiliations or financial involvement with any organization or entity with a financial interest in or financial conflict with the subject matter or materials discussed in the manuscript. This includes employment, consultancies, honoraria, stock ownership or options, expert testimony, grants or patents received or pending, or royalties.

No writing assistance was utilized in the production of this manuscript.

\section{References}

1. Valizadeh A, Mikaeili H, Samiei M et al. Quantum dots: synthesis, bioapplications, and toxicity. Nanoscale Res. Lett. 7(1), 480 (2012).

2. Walling MA, Novak JA, Shepard JR. Quantum dots for live cell and in vivo imaging. Int. J. Mol. Sci. 10(2), 441-491 (2009).

3. Nigam P, Waghmode S, Louis M, Wangnoo S, Chavan P, Sarkar D. Graphene quantum dots conjugated albumin nanoparticles for targeted drug delivery and imaging of pancreatic cancer. J. Mater. Chem. B 2(21), 3190-3195 (2014).

4. Chen L, Willoughby A, Zhang J. Luminescent gelatin nanospheres by encapsulating CdSe quantum dots. Luminescence 29(1), 74-78 (2014).

5. Chu M, Wu F, Zhang Q et al. A novel method for preparing quantum dot nanospheres with narrow size distribution. Nanoscale 2(4), 542-547 (2010).

6. Aswathy RG, Sivakumar B, Brahatheeswaran D et al. Biocompatible fluorescent zein nanoparticles for simultaneous bioimaging and drug delivery application. Adv. Nat. Sci. Nanosci. Nanotech. 3(2), 025006 (2012).

7. Wong C, Stylianopoulos T, Cui J et al. Multistage nanoparticle delivery system for deep penetration into tumor tissue. Proc. Natl Acad. Sci. USA 108(6), 2426-2431 (2011).

8. Bwatanglang IB, Mohammad F, Yusof NA et al. Folic acid targeted Mn: ZnS quantum dots for theranostic applications of cancer cell imaging and therapy. Int. J. Nanomed. 11, 413-428 (2016).

9. Kim KS, Hur W, Park SJ et al. Bioimaging for targeted delivery of hyaluronic acid derivatives to the livers in cirrhotic mice using quantum dots. ACS Nano 4(6), 3005-3014 (2010).

10. Kim KS, Kim S, Beack S, Yang JA, Yun SH, Hahn SK. In vivo real-time confocal microscopy for target-specific delivery of hyaluronic acid-quantum dot conjugates. Nanomedicine 8(7), 1070-1073 (2012).

11. Abdelhamid AS, Helmy MW, Ebrahim SM et al. Layer-by-layer gelatin/chondroitin quantum dots-based nanotheranostics: combined rapamycin/celecoxib delivery and cancer imaging. Nanomedicine (Lond.) 13(14), 1707-1730 (2018).

12. Abdelhamid AS, Zayed DG, Helmy MW et al. Lactoferrin-tagged quantum dots-based theranostic nanocapsules for combined COX-2 inhibitor/herbal therapy of breast cancer. Nanomedicine (Lond.) 13(20), 2637-2656 (2018).

13. Volsi AL, Fiorica C, D’amico M et al. Hybrid Gold/Silica/Quantum-dots supramolecular-nanostructures encapsulated in polymeric micelles as potential theranostic tool for targeted cancer therapy. Eur. Polym. J. 105, 38-47 (2018).

14. Wang Y, Wang Y, Chen G, Li Y, Xu W, Gong S. Quantum-dot-based theranostic micelles conjugated with an anti-EGFR nanobody for triple-negative breast cancer therapy. ACS Appl. Mater. Interfaces 9(36), 30297-30305 (2017).

15. Olerile LD, Liu Y, Zhang B et al. Near-infrared mediated quantum dots and paclitaxel co-loaded nanostructured lipid carriers for cancer theragnostic. Colloids Surf. B Biointerfaces 150, 121-130 (2017).

16. Al-Jamal WT, Al-Jamal KT, Cakebread A, Halket JM, Kostarelos K. Blood circulation and tissue biodistribution of lipid-quantum dot (L-QD) hybrid vesicles intravenously administered in mice. Bioconjug. Chem. 20(9), 1696-1702 (2009).

17. Weng KC, Hashizume R, Noble CO et al. Convection-enhanced delivery of targeted quantum dot-immunoliposome hybrid nanoparticles to intracranial brain tumor models. Nanomedicine (Lond.) 8(12), 1913-1925 (2013).

18. Chen T, Zhao T, Wei D, Wei Y, Li Y, Zhang H. Core-shell nanocarriers with ZnO quantum dots-conjugated Au nanoparticle for tumor-targeted drug delivery. Carbohydr. Polym. 92(2), 1124-1132 (2013).

19. Yang W, Guo W, Gong X et al. Facile synthesis of Gd-Cu-In-S/ZnS bimodal quantum dots with optimized properties for tumor targeted fluorescence/MR in vivo imaging. ACS Appl. Mater. Interfaces 7(33), 18759-18768 (2015).

20. Ou J, Wang F, Huang $\mathrm{Y}$ et al. Fabrication and cyto-compatibility of $\mathrm{Fe}_{3} \mathrm{O}_{4} / \mathrm{SiO}_{2} /$ graphene-CdTe QDs/CS nanocomposites for drug delivery. Colloids Surf. B Biointerfaces 117, 466-472 (2014). 